\title{
Association Pyoderma Gangrenosum and Systemic Lupus Erythematosus
}

\author{
A Rkiouak, PhD", I El Kassimi, MD, N Sahel, MD, M Zaizae, MD and Y Sekkach, PhD
}

Department of Internal Medicine A, Mohammed V Military Hospital, Medical School of Rabat, Morocco

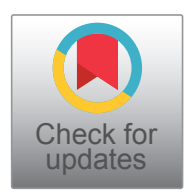

*Corresponding author: Adil Rkiouak, PH.D, Department of Internal Medicine A, Mohammed V Military Hospital, Medical School of Rabat, Morocco, Tel: +212-66-179-44-04, Fax: +212-66-179-44-04

\section{Introduction}

19-year-old patient, follow-up for 8 years for SLE treated with hydroxychloroquine $400 \mathrm{mg} /$ day and who had been in remission for a year. SLE diagnosis was based on the association of a malar rash, photosensitivity, lymphopenia, autoimmune hemolytic anemia, antinuclear antibodies positivity, anti-DNA antibodies identified and lupus nephritis.

On December 2019, patient noticed a well-defined irregular popular lesion on left leg (Figure 1) associated with ulcerations appeared 1 month later. Treatment with local antibiotics was without improvement.

Both lesions had well-defined purplish undermined borders and a necrotic base with surrounding erethey$\mathrm{ma}$, and there was marked tenderness on palpation.

Skin examination found a deep ulceration making 3 $\mathrm{cm}$ of diameter of the anterior face left leg with inflammatory border.

A Doppler ultrasound of right leg showed no evidence of venous obstruction. An arterial sonography scan showed no evidence of arterial insufficiency.

Histologic examination of the lesion showed a dense infiltration of dermis by leukocytoclastic or intact neutrophils, without granuloma or vascular damage. The diagnosis of ulcerative variant of PG was retained concluding in pyoderma gangrenosum. No pathogens including atypical mycobacteria or yeast were evidenced.

A treatment with prednisone $1 \mathrm{mg} / \mathrm{kg} /$ day orally was started up, and complete resolution of PG was obtained within 6 months (Figure 2). The patient did not present any recurrence of PG to date, with a 1-year follow-up.

Pyoderma Gangrenosum (PG) is part of the spectrum of the neutrophilic dermatoses, which is usually idiopathic. PG typically presents with single or multiple skin ulcers with undetermined erythematous-violaceous borders.

Although PG has been associated with several systemic diseases, it has rarely been reported in association with Systemic Lupus Erythematosus (SLE).

No absolute criteria for the diagnosis of PG are available, but diagnostic criteria, to which our patient replied, have been proposed [1].

The diagnosis of PG was made based on features of rapid onset and progression, resistance to treatment with antibiotics, characteristic of lesion (ulcerating, well-defined purple undermined borders) and histological study.

More than $50 \%$ of patients with PG have an associated systemic disease, most commonly inflammatory bowel disease (14-34\%), arthropathies (11-25\%) and hematologic disease or hematologic malignancy $(20 \%)$ [2].

Systemic Lupus Erythematosus (SLE) is not a commonly recognized associate of PG.

This association may be supported by common innate immunity dysregulation and abnormal neutrophil activation.

Among the reported cases of patients with PG in a context of SLE, a large majority were women $[3,4]$. 


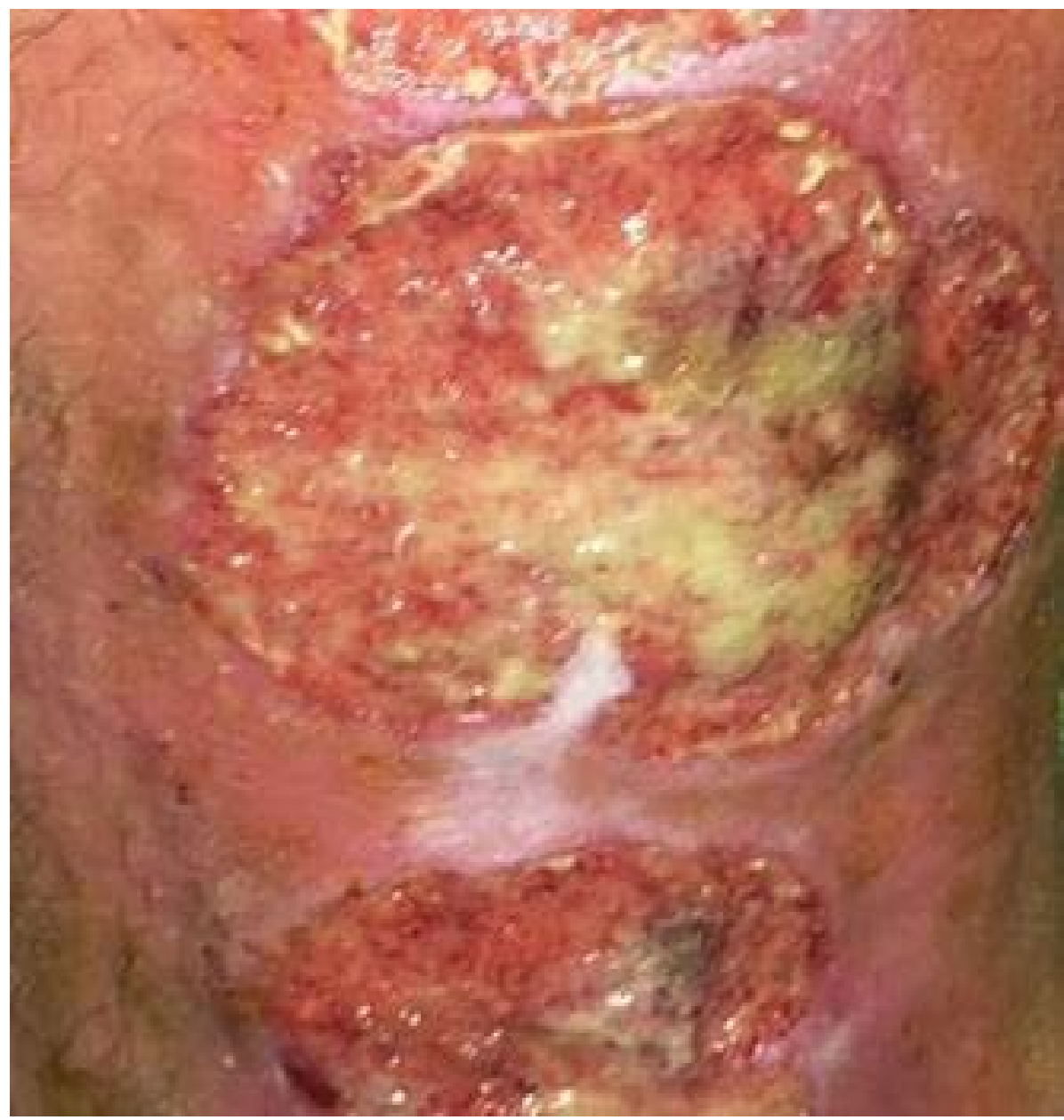

Figure 1: Deep ulcerations with inflammatory border of the left leg.

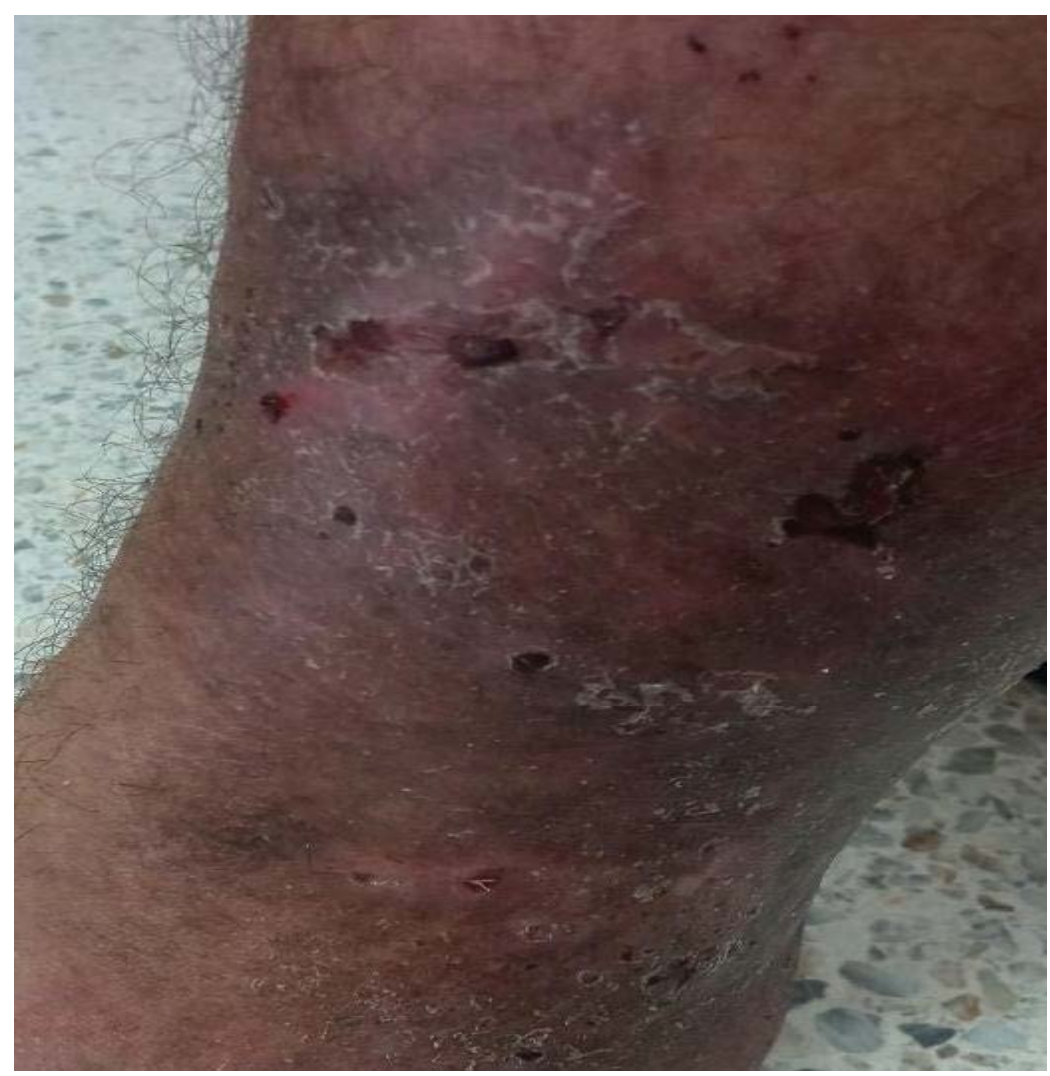

Figure 2: Healing ulcerations after corticosteroid therapy. 
The diagnosis of lupus usually precedes the onset of $P G$, as in our patient.

Classically, the treatment of PG is based on (colchicine, dapsone, corticosteroids, minocycline). However, SLE treatments (hydroxychloroquine, methotrexate, mycophenolate mofetil, and cyclophosphamide) appear to be effective in PG. Our patient was treated with general corticosteroid therapy with regression of lupus lesions and PG lesions.

The association between LES and PG is rare. The prognosis of the disease does not appear to be worsen by PG. corticosteroid therapy is usually sufficient to treat a possible association.

\section{References}

1. Maverakis E, Ma C, Shinkai K, Fiorentino D, Callen JP, et al. (2018) Diagnostic Criteria of Ulcerative Pyoderma Gangrenosum. JAMA Dermatology 154: 461-466.

2. Binus AM, Qureshi AA, Li VW, Winterfield LS (2011) Pyoderma gangrenosum: a retrospective review of patient characteristics, comorbidities and therapy in 103 patients. Br J Dermatol 165: 1244-1250.

3. Gonzalez-Moreno J, Ruiz-Ruigomez M, Callejas Rubio JL,Ríos Fernández R, Ortego Centeno N, et al. (2015) Pyoderma gangrenosum and systemic lupus erythematosus: a report of five cases and review of the literature. Lupus 24: 130-137.

4. Hamzi AM, Bahadi A, Alayoud A, El Kabbaj D, Benyahia M (2013) Skin ulcerations in a lupus hemodialysis patient with hepatitis C infection: what is your diagnosis? Iran J Kidney Dis 7: 191. 